\title{
MicroRNA-3653 inhibits the growth and metastasis of hepatocellular carcinoma by inhibiting ITGB1
}

\author{
LIJUAN ZHANG, TAO ZHANG, ZERUN DENG and LIHUA SUN \\ The Infectious Disease Center, The First Affiliated Hospital of Xinjiang Medical University, \\ Urumqi, Xinjiang 830054, P.R. China
}

Received July 15, 2018; Accepted December 5, 2018

DOI: $10.3892 /$ or.2019.6971

\begin{abstract}
RNAs (miRNAs) play critical roles in hepatocellular carcinoma (HCC). However, the expression and biological function of miR-3653 in HCC remain unknown. The present study demonstrated that miR-3653 expression was significantly decreased in HCC tissues and cells using qRT-PCR. A decreased miR-3653 level was associated with unfavorable clinical features and poor prognosis of HCC patients. MTT, BrdU, Transwell and western blot assays showed that miR-3653 overexpression inhibited the growth, migration, invasion and epithelial-mesenchymal transition (EMT) of HCCLM3 cells while its knockdown promoted the growth and metastatic ability of Hep3B cells. In vivo experiments showed that miR-3653 overexpression inhibited the subcutaneous and the lung metastasis of HCCLM3 cells in nude mice. Mechanistically, integrin- $\beta 1$ (ITGB1) was identified to be the downstream target of miR-3653 in HCC. ITGB1 overexpression reversed the inhibitory effects of miR-3653 on the growth, metastasis and EMT of HCCLM3 cells.
\end{abstract}

\section{Introduction}

MicroRNAs (miRNAs) are a cluster of short non-coding RNAs ( 20 nucleotides long) (1). miRNAs can post-transcriptionally regulate gene expression by interacting with the $3^{\prime}$ untranslated region (3'-UTR) of targeted messenger RNAs (mRNAs). Functionally, miRNAs were found to play critical roles in various human diseases including human cancers. Numerous miRNAs have been found to be aberrantly expressed in human cancers and have been proposed as promising biomarkers and prognostic predictors for cancer patients (2). Among these cancer-associated miRNAs, miR-3653 is a novel cancer-associated miRNA. miRNA expression profiles showed that miR-3653 is aberrantly

Correspondence to: Professor Tao Zhang, The Infectious Disease Center, The First Affiliated Hospital of Xinjiang Medical University, 137 South Lei Yushan Road, Urumqi, Xinjiang 830054, P.R. China E-mail: hangt7711_vip@yeah.net

Key words: miR-3653, hepatocellular carcinoma, ITGB1, growth, metastasis expressed in lung adenocarcinoma (3) and colon cancer (4). A decreased expression level of $\mathrm{miR}-3653$ was found to be correlated with a poor prognosis of lung adenocarcinoma patients.

Hepatocellular carcinoma (HCC) is a fatal malignancy affecting millions of individuals worldwide. HCC ranks as the second leading cause of cancer-related death (5). The overall survival for HCC patients is unsatisfactory, and the 5-year survival rate of HCC patients is less than 40\%; and even worse for those in advanced stages (6). Uncontrolled growth and occurrence of local and systemic metastasis are the main reasons for the poor prognosis of HCC patients. During the last two decades, increasing studies have shown that miRNAs play critical roles in the progression of HCC. miRNAs were found to regulate the growth, metastasis and drug resistance of HCC cells. However, the expression and biological functions of miR-3653 in HCC remain unknown.

The present study demonstrated that compared with adjacent non-tumor tissues, miR-3653 expression was significantly decreased in HCC tissues. Patients with a low miR-3653 level showed decreased overall and disease-free survival. Decreased expression of miR-3653 was found to be associated with unfavorable clinicopathological features (large tumor size and occurrence of metastasis) of HCC patients. Using gain- and loss-of-function assays, miR-3653 was found to inhibit the growth and metastasis of HCC cells. In vivo assays showed that miR-3653 slowed down the subcutaneous growth and reduced the lung metastasis of HCC cells in nude mice. Mechanistically, the present study revealed that integrin- $\beta 1$ (ITGB1) was the downstream target of miR-3653 in HCC cells. Moreover, we demonstrated that targeting ITGB1 was critical for the biological functions of miR-3653 in HCC.

\section{Materials and methods}

Clinical tissues. HCC tissues along with adjacent non-tumor tissues were collected from $60 \mathrm{HCC}$ patients $(37$ male and 23 female patients, average age $43.9 \pm 9.7$ years) who received surgical treatment at The Infectious Disease Center, The First Affiliated Hospital of Xinjiang Medical University (Urumqi, Xinjiang) from January 2002 to December 2010. All clinical tissues were pathologically confirmed as HCC and maintained at $-80^{\circ} \mathrm{C}$ before being subjected to further experiments. Written informed consent was obtained from every patient enrolled in 
this study. Ethical protocols for using HCC patient samples were approved by the Institutional Research Ethics Committee of the First Affiliated Hospital of Xinjiang Medical University (Urumqi, China).

Cell culture. HCC cell lines including Hep3B, Huh7, MHCC97H and HCCLM3 and the immortalized hepatocyte L-02 cell line were obtained from the Cell Bank of the Chinese Academy of Sciences (Shanghai, China). Dulbecco's modified Eagle's medium (DMEM; Gibco; Thermo Fisher Scientific, Inc., Waltham, MA, USA) along with $10 \%$ fetal bovine serum (10\%) (FBS; Gibco; Thermo Fisher Scientific, Inc.) was used for cell culture. Cell cultures were maintained in a cell incubator at $37^{\circ} \mathrm{C}$ with $5 \% \mathrm{CO}_{2}$.

Transfection of HCC cells. Transfection of HCC cells was performed using Lipofectamine 2000 (Invitrogen; Thermo Fisher Scientific, Inc.) based on the manufacturer's instructions. miR-3653 mimic (50 nM; product no. HMI0001-HMI2785) and non-targeting control (50 nM; product no. HMC0002) were obtained from Sigma-Aldrich (Merck KGaA, Darmstadt, Germany), and transfected into HCCLM3 cells. miR-3653 inhibitor (50 nM; product no. HSTUD1287) and the corresponding negative control (50 nM; product no. NCSTUD001) were obtained from Sigma-Aldrich (Merck KGaA) and transfected into Hep3B cells. The vector used for overexpression of ITGB1 was pcDNA 3.1 which was obtained from Addgene (Cambridge, MA, USA). ITGB1 vector $(1.5 \mu \mathrm{g} / \mathrm{ml}$; cat. no. 51920) and the empty vector $(1.5 \mu \mathrm{g} / \mathrm{ml}$; cat. no. 52535$)$ were obtained from Addgene and co-transfected with miR-3653 mimic or non-targeting control into HCCLM3 cells: HCCLM3 cells co-transfected with non-targeting control (product no. HMC0002) and control vector (cat. no. 52535), HCCLM3 cells transfected with miR-3653 mimic (product no. HMI0001-HMI2785) and control vector (cat. no. 52535), and HCCLM3 cells transfected with miR-3653 mimic (product no. HMI0001-HMI2785) and ITGB1 vector (cat. no. 51920). Forty-eight hours after the cellular transfection, these cells were collected for western blot analysis, qRT-PCR, MTT, BrdU and Transwell assays, and in vivo experiments. The efficacy of cell transfection were confirmed by qRT-PCR or western blot analysis.

Quantitative real-time reverse transcription- $P C R$ ( $q R T-P C R)$. RNA in clinical tissues and HCC cells were extracted using TRIzol and RNeasy Mini kit (Qiagen, Shanghai, China). The Transcriptional First Strand cDNA Synthesis kit and SYBR-Green PCR Master Mix (Applied Biosystems, Foster City, CA, USA) were used for reverse transcription reactions and quantitative real-time PCR. Primers for E-cadherin, N-cadherin, ITGB1, GAPDH, miR-3653 and U6 were obtained from Guangzhou GeneCopoeia (Guangzhou, China). GAPDH was used as the internal controls for E-cadherin, $\mathrm{N}$-cadherin and ITGB1. U6 was used as the internal controls for miR-3653. Primer sequences were listed as below: miR-3653 forward, 5'-TCTCCCGAGAGACATATTT-3' and reverse, 5'-GATGAG AAGGTATGAATCA-3'; U6 forward, 5'-GCTTCGGCAGCA CATATACTAAAAT-3' and reverse, 5'-CGCTTCACGAAT TTGCGTGTCAT-3'; E-cadherin forward, 5'-CAGCATCAC TGGCCAAGGAGCTGA-3' and reverse, 5'-GACCACACT
GATGACTCCTGTGTTCC-3'; N-cadherin forward, 5'-GTC ATCTTGATCTCATAACGCTGG-3' and reverse, 5'-AGC CCATCTGTACCTGTGGTTCA-3'; ITGB1 forward, 5'-TCA GAATTGGATTTGGCTCATTT-3' and reverse, 5'-CCTGAG CTTAGCTGGTGTTGTG-3'; GAPDH forward, 5'-GGTCAC CAGGGCTGCTTTTA-3' and reverse, 5'-GGATCTCGCTCC TGGAAGATG-3'. The relative expression levels of miRNAs or mRNAs were determined using $\Delta \Delta \mathrm{Cq}$-based fold-change calculations as previously described $(7,8)$.

Western blot analysis. Proteins in clinical tissues and HCC cells were extracted using RIPA buffer and subjected to concentration measurements using BCA kit. After being separated in SDS-PAGE gels, the protein $(20 \mu \mathrm{g})$ on SDS-PAGE gels (4-20\%) were transferred to polyvinylidene fluoride membrane. These membranes were incubated with 5\% non-fat dry milk (diluted in TBST) at room temperature for $1 \mathrm{~h}$ and primary antibodies of E-cadherin (dilution 1:1,000; cat. no. 3195; Cell Signaling Technologies, Inc., Danvers, MA, USA), N-cadherin (dilution 1:500; cat. no. 4061; Cell Signaling Technologies, Inc.), ITGB1 (dilution 1:1,000; cat. no. 4706; Cell Signaling Technologies, Inc.) and GAPDH (dilution 1:2,000; cat. no. sc-32233; Santa Cruz Biotechnology, Inc., Santa Cruz, CA, USA) overnight at $4^{\circ} \mathrm{C}$. GAPDH was used as internal control. Protein signals were detected using ECL reagents (Amersham Biosciences; GE Healthcare, Chicago, IL, USA).

MTT and BrdU assays. For MTT assay, HCCLM3 cells overexpressing miR-3653 or those in the control group, and Hep3B cells with miR-3653 knockdown or those in the negative control group (5,000 cells/well) were seeded into 96-well plates. Twenty-four, 28 and $72 \mathrm{~h}$ after cell seeding, MTT (Sigma-Aldrich; Merck KGaA) was added into each well and incubation was carried out for $4 \mathrm{~h}$ at $37^{\circ} \mathrm{C}$. Absorbance at $490 \mathrm{~nm}$ was measured as the indicator of cell viability. For BrdU assay, HCC cells described above were stained with bromodeoxyuridine for $1 \mathrm{~h}$ at room temperature and then incubated with anti-BrdUrd antibody (Sigma-Aldrich; Merck KGaA). BrdU-positive cells were counted under fluorescence microscope (Axioskop 2 Plus; Carl Zeiss Co., Ltd., Jena, Germany).

Transwell assay. The migratory and invasive abilities of HCC cells were assessed by Transwell assay. The day before Transwell assay, HCCLM3 cells overexpressing miR-3653 or those in the control group, and Hep3B cells with miR-3653 knockdown or those in the negative control group were starved in serum-free DMEM media overnight. Then, the cells $\left(3 \times 10^{4}\right)$ resuspended in $200 \mu$ l basal DMEM were added to the upper chamber of a Transwell insert. A total of $600 \mu 1$ serum-containing DMEM (20\% FBS) used as chemoattractant was added to the lower chamber. For the invasion experiments, $100 \mu 1$ Matrigel (diluted in DMEM at the ratio of 1:8) was added to the bottom of the upper chamber. Twenty-four hours later, cells that had migrated through the membrane were stained with crystal violet. The number of migrated or invaded cells was counted under a light microscope at x40 magnification.

Luciferase assay. 3'-UTR of ITGB1 containing the binding sequences for miR-3653 or the mutated 3'-UTR of ITGB1 

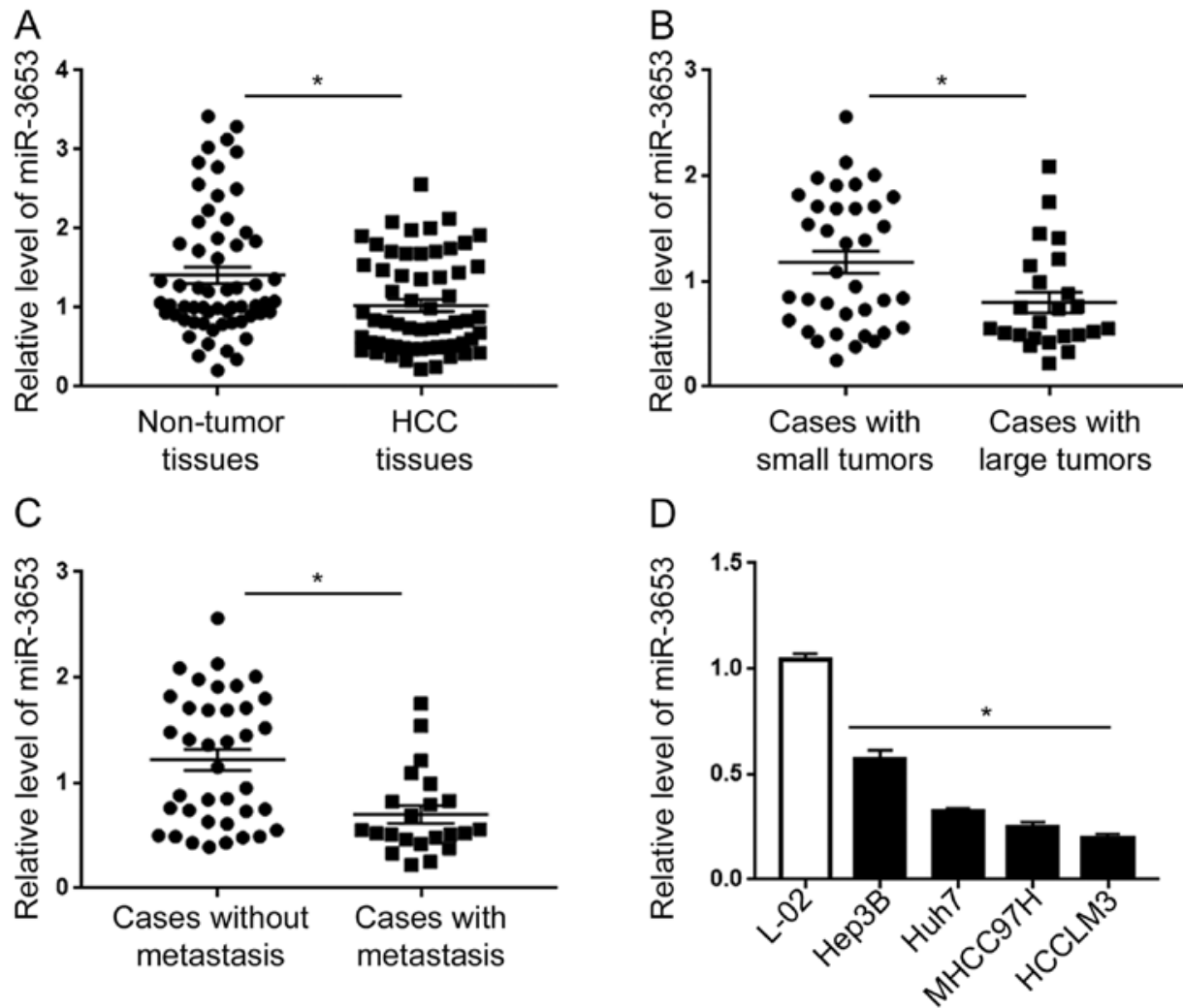

Figure 1. Expression of miR-3653 in HCC tissues and cells. (A) The expression level of miR-3653 was compared between HCC tissues and adjacent non-tumor tissues. (B) The expression level of miR-3653 was compared between patients with large $(>5 \mathrm{~cm})$ and small $(\leq 5 \mathrm{~cm})$ tumors. (C) The expression level of miR-3653 was compared between patients with and without metastasis. (D) The expression of miR-3653 was evaluated in 4 HCC cell lines (Hep3B, Huh7, MHCC 97H and HCCLM3) and immortalized hepatocyte L-02 cells. ${ }^{*} \mathrm{P}<0.05$. HCC, hepatocellular carcinoma.

was used to construct the wild-type ITGB1-3'-UTR or mutant ITGB1-3'-UTR. HCC cells in 12-well plates were transfected with wild-type or mutant 3'-UTR of ITGB1 along with miR-3653 mimic or inhibitor or the corresponding control vector. After co-transfection, the luciferase activity was measured by luciferase reporter kit (Promega Corporation, Madison, WI, USA).

In vivo tumor growth and metastasis assay. For the in vivo tumor growth studies, nude mice (6-weeks of age, 4 female mice each group) were injected with HCCLM3 cells overexpressing miR-3653 or cells in the control group $\left(1 \times 10^{6}\right)$. The length and width of tumor nodules were measured every 7 days. After 28 days, the nude mice were sacrificed using cervical dislocation and the subcutaneous tumors were removed and subjected to measurement of the volume (volume $=$ width $^{2} \mathrm{x}$ length/2). For tail vein injection model, HCCLM3 cells overexpressing miR-3653 or cells in the control group $\left(5 \times 10^{4}\right)$ were injected into nude mice (4 mice each group) through the tail vein. All mice (Saierbio, Tianjing, China), with 4 female mice in each group, were maintained in specific pathogen-free (SPF) rooms under a 12-h light/dark cycle, with two mice in each static filter-topped cage. The initial weight of these mice were approximately $20 \mathrm{~g}$. Eight weeks after tail vein injection, these nude mice were sacrificed using cervical dislocation and the lungs were removed and subjected to H\&E staining. The protocols regarding the in vivo manipulations were approved by the Animal Care Committee of the First Affiliated Hospital of Xinjiang Medical University.
Statistical analysis. All quantitative data are presented as the mean \pm standard error of the mean (SEM) from at least three independent replicates. SPSS software 13.0 (SPSS, Inc., Chicago, IL, USA) was used for statistical analysis, a two-tailed Student t-test and Kaplan-Meier analysis were used to analyze the differences between two groups. One-way analysis of variance (ANOVA) was used to examine the statistical difference between multiple groups with least significant difference (LSD) test as post hoc test. Differences between groups were considered statistically significant at $\mathrm{P}<0.05$.

\section{Results}

miR-3653 expression is decreased in HCC tissues and cells. qRT-PCR of the clinical specimens showed that compared with non-tumor tissues, HCC tissues showed a significantly decreased level of miR-3653 ( $\mathrm{P}<0.05$; Fig. 1A). Subgroup analysis showed that compared with tumors $>5 \mathrm{~cm}$, small tumors tumors $\leq 5 \mathrm{~cm}$ showed significantly increased level of miR-3653 ( $\mathrm{P}<0.05$; Fig. 1B). Moreover, patients with local or systemic metastasis showed a significantly decreased level of miR-3653 than those without metastasis $(\mathrm{P}<0.05$; Fig. 1C). Finally, we compared the expression level of miR-345 in HCC cell lines and L-02 cells. Compared with that in L-02 cells, the level of miR-3653 in the four HCC cell lines was significantly decreased $(\mathrm{P}<0.01$; Fig. 1D). Among the four HCC cell lines, the expression level of miR-3653 was highest in the Hep3B cells and lowest in the HCCLM3 cells. Furthermore, we investigated the association between the miR-3653 level and patient 
A

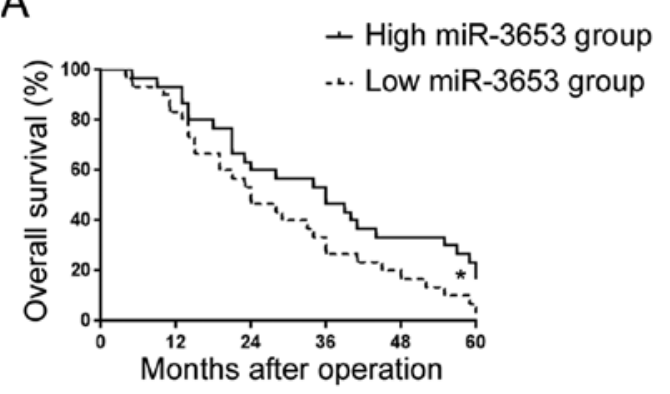

$\mathrm{B}$

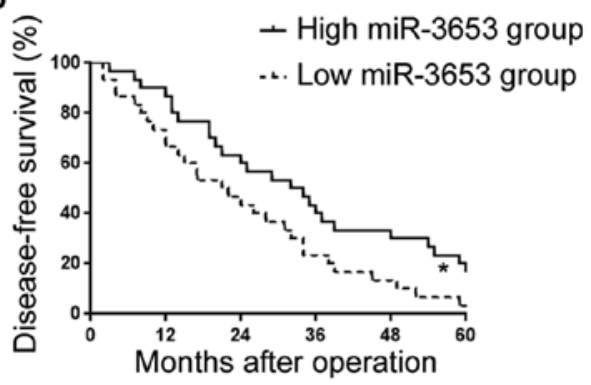

Figure 2. The prognostic value of miR-3653 in HCC patients. (A) Overall survival and (B) disease-free survival was compared between patients with a high miR-3653 level and those with a low miR-3653 level. ${ }^{*} \mathrm{P}<0.05$. HCC, hepatocellular carcinoma.

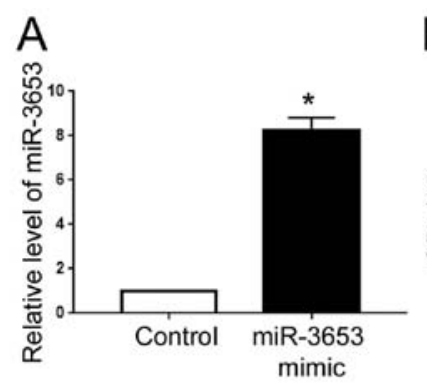

D
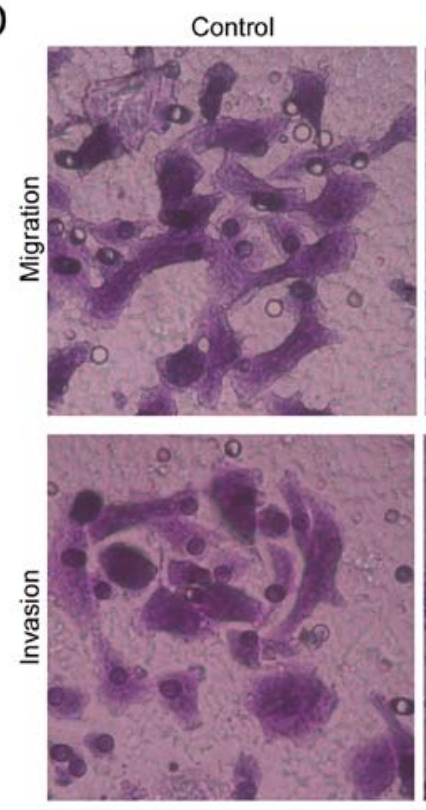

B

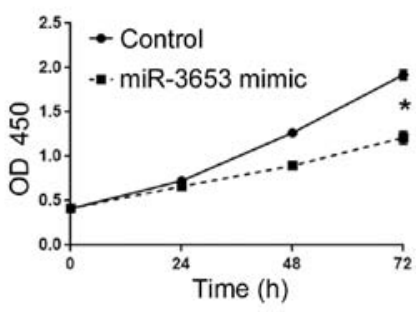

miR-3653 mimic
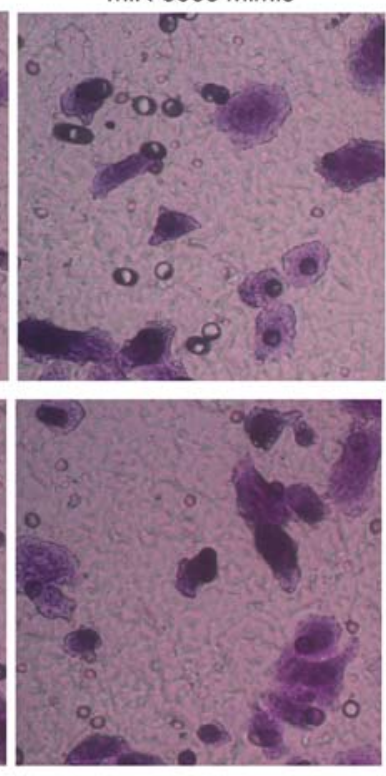

C

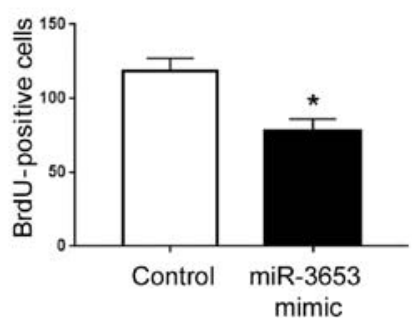

mimic
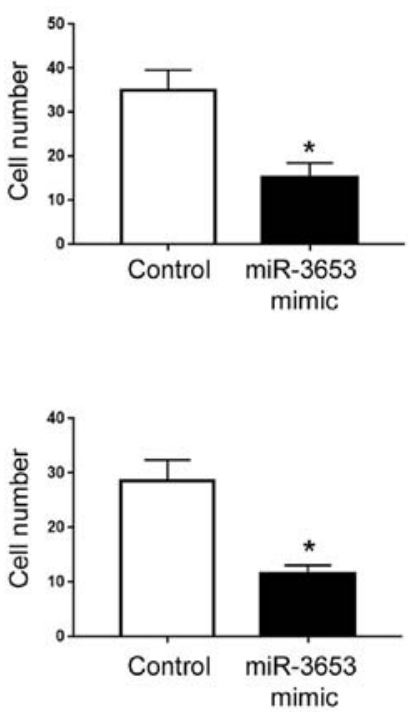

Figure 3. miR-3653 overexpression inhibits the growth and metastatic ability of HCCLM3 cells. (A) Transfection of miR-3653 mimics significantly increased the expression level of miR-3653 in HCCLM3 cells. (B) miR-3653 overexpression decreased the cell viability of HCCLM3 cells, as suggested by MTT assay. (C) miR-3653 overexpression decreased the cell proliferation of HCCLM3 cells, as suggested by BrdU assay. (D) miR-3653 overexpression inhibited the migration and invasion of HCCLM3 cells, as suggested by Transwell assays. " $\mathrm{P}<0.05$.

prognosis. Kaplan-Meier survival analysis showed that patients with a low miR-3653 level had significantly decreased overall survival $(\mathrm{P}<0.05$; Fig. $2 \mathrm{~A})$ and disease-free survival $(\mathrm{P}<0.05$; Fig. 2B).

miR-3653 inhibits the growth and metastatic ability of HCC cells in vitro. To determine the biological functions of miR-3653 in HCC cells, we performed gain- and loss-of function experiments in HCCLM3 and Hep3B cells, respectively. Transfection of miR-3653 mimics into HCCLM3 cells effectively increased the
miR-3653 level ( $\mathrm{P}<0.05$; Fig. 3A). MTT and BrdU assays showed that overexpression of miR-3653 led to decreased cell viability and proliferation of HCCLM3 cells ( $\mathrm{P}<0.05$; Fig. 3B and $\mathrm{C}$ ). Transwell assay showed that forced expression of miR-3653 led to significantly decreased ability of cell migration and invasion $(\mathrm{P}<0.05$; Fig. 3D). In contrast, we performed miR-3653 knockdown in Hep3B cells. miR-3653 inhibitor significantly decreased the miR-3653 level in Hep3B cells ( $\mathrm{P}<0.05$; Fig. 4A), and led to increased cell viability, proliferation, migration and invasion $(\mathrm{P}<0.05$; Fig. 4B-D). 

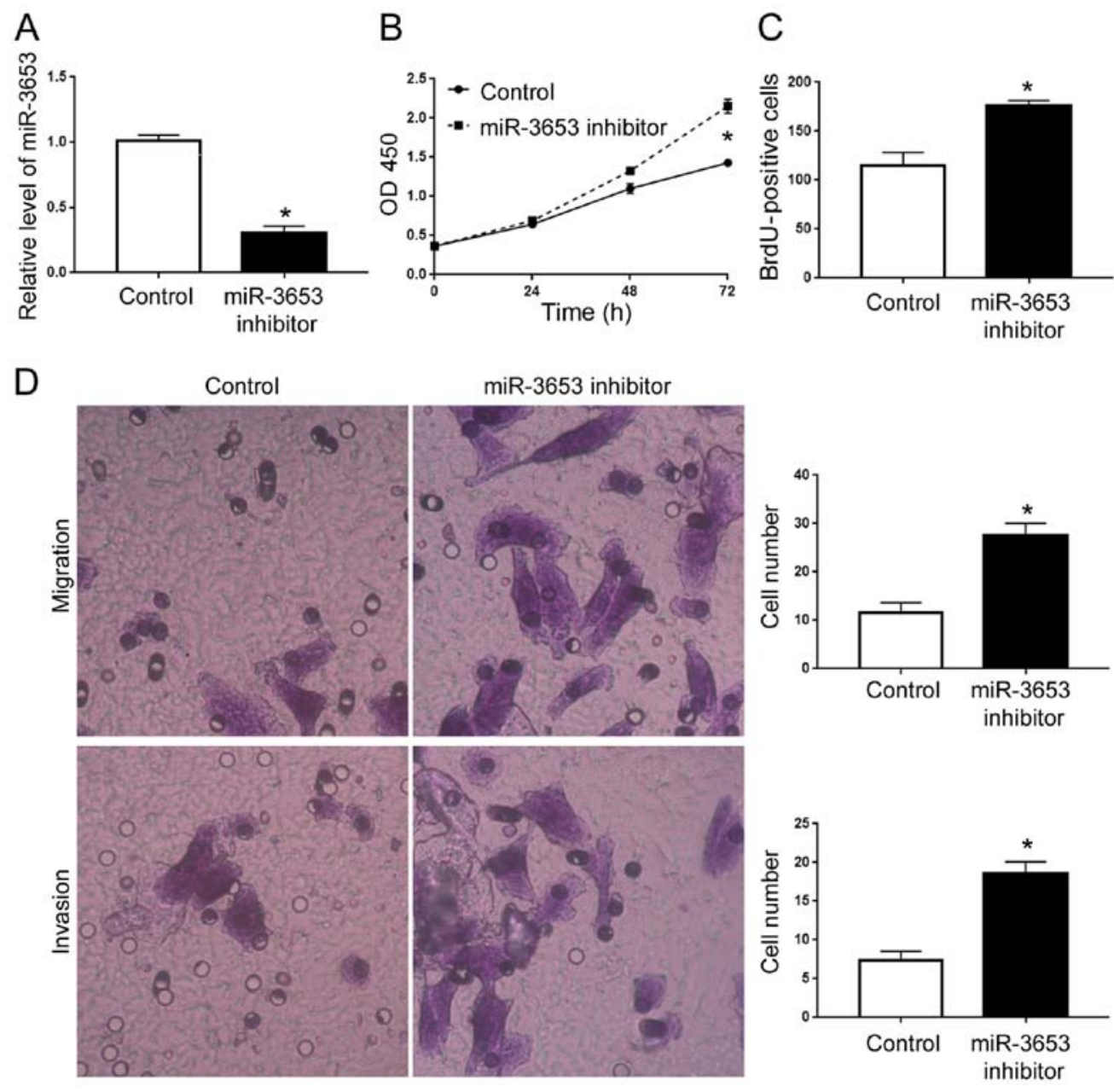

Figure 4. miR-3653 knockdown increases the growth and metastatic ability of Hep3B cells. (A) Transfection of miR-3653 inhibitor significantly decreased the expression level of miR-3653 in Hep3B cells. (B) miR-3653 knockdown increased the cell viability of Hep3B cells, as suggested by MTT assay. (C) miR-3653 knockdown increased the cell proliferation of Hep3B cells, as suggested by BrdU assay. (D) miR-3653 knockdown promoted the migration and invasion of Hep3B cells, as suggested by Transwell assays. "P<0.05.

miR-345 inhibits the EMT of HCC cells. EMT has been widely accepted as a critical mechanism for cancer metastasis (9). Therefore, we evaluated the EMT status after altering the miR-3653 level in HCCLM3 and Hep3B cells. qRT-PCR showed that overexpression of miR-3653 increased E-cadherin mRNA and decreased N-cadherin mRNA $(\mathrm{P}<0.05$; Fig. 5A). Western blot analysis showed that forced expression of miR-3653 led to elevated E-cadherin protein and decreased $\mathrm{N}$-cadherin protein $(\mathrm{P}<0.05$; Fig. $5 \mathrm{~B})$. In contrary, miR-3653 knockdown decreased the mRNA and protein level of E-cadherin ( $\mathrm{P}<0.05$; Fig. 5C and $\mathrm{D})$ and increased $\mathrm{N}$-cadherin expression $(\mathrm{P}<0.05$; Fig. $5 \mathrm{C}$ and $\mathrm{D})$.

miR-3653 inhibits the growth and metastasis of HCCLM3 cells in nude mice. After confirming the in vitro influence of miR-3653 on HCC cells, we further assessed the in vivo function of miR-3653 in HCC. Subcutaneous injection of HCCLM3 cells with or without miR-3653 overexpression showed that miR-3653 significantly slowed down the growth of HCCLM3 cell-derived tumors in nude mice $(\mathrm{P}<0.05$; Fig. $6 \mathrm{~A})$. Tail vein injection model showed that overexpression of miR-3653 effectively reduced the number of lung metastatic nodules in nude mice $(\mathrm{P}<0.05$; Fig. 6B).
ITGB1 is the downstream target of miR-3653 in HCC. We further searched the public database TargetScan (http://www.targetscan.org) to identify potential downstream targets of miR-3653. ITGB1, a well-known oncogenic protein which plays a critical role in HCC progression (10), was one of the predicted downstream targets of miR-3653. As shown in Fig. 7A, ITGB1 3'-UTR contained the binding sequences for miR-3653. Luciferase assay showed that overexpression of miR-3653 decreased the luciferase activity of wild-type ITGB1 3'-UTR while miR-3653 knockdown increased that of ITGB1 3'-UTR (P<0.05; Fig. 7B). Neither miR-3653 overexpression nor miR-3653 knockdown affected the luciferase of mutated ITGB1 3'-UTR (Fig. 7B). qRT-PCR and western blot analysis showed that miR-3653 overexpression decreased the mRNA and protein level of ITGB1 in HCCLM3 cells $(\mathrm{P}<0.05$; Fig. 7C and D). Knockdown of miR-3653 increased the expression of ITGB1 in Hep3B cells $(\mathrm{P}<0.05$; Fig. 7E and F). To further confirm the regulatory effect of miR-3653 on ITGB1, we detected the ITGB1 expression in HCC tissues. Compared with those with high miR-3653 level, tissues with low miR-3653 level showed increased expression of ITGB1. The IHC score of ITGB1 in HCC tissues with low miR-3653 level was significantly higher than that in tissues with high miR-3653 level $(\mathrm{P}<0.05$; Fig. 7G). 


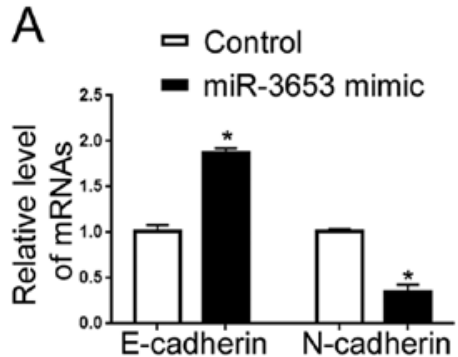

C

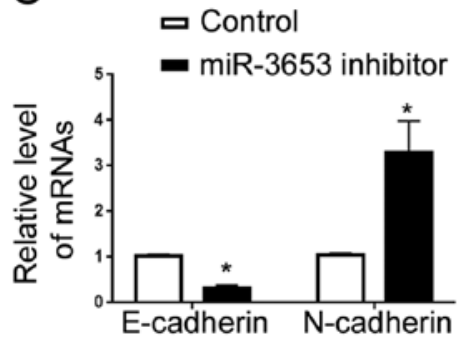

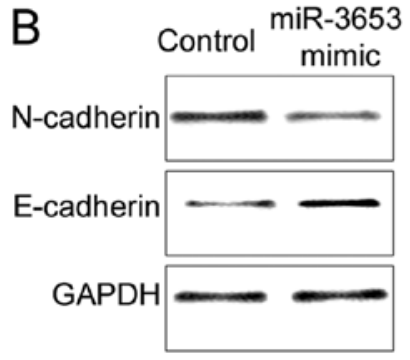

D

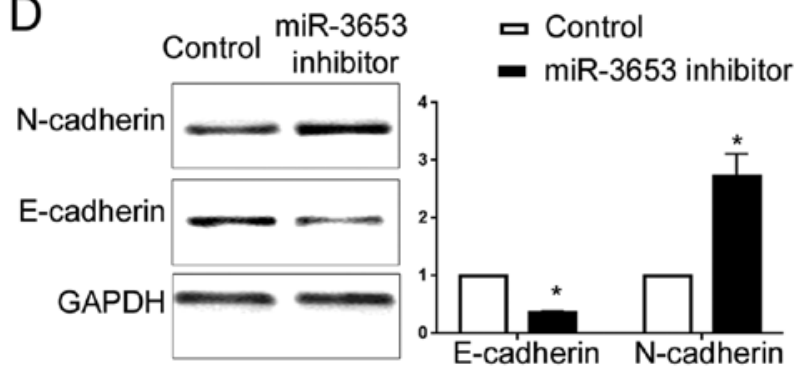

Figure 5. miR-3653 inhibits the EMT in HCC cells. (A) Overexpression of miR-3653 led to an increased level of E-cadherin mRNA and decreased level of $\mathrm{N}$-cadherin mRNA in HCCLM3 cells, as suggested by qRT-PCR. (B) miR-3653 overexpression led to an increased level of E-cadherin protein and decreased level of N-cadherin protein in HCCLM3 cells, as suggested by western blot analysis. (C) Knockdown of miR-3653 led to decreased level of E-cadherin mRNA and increased level of N-cadherin mRNA in Hep3B cells, as suggested by qRT-PCR. (D) miR-3653 knockdown led to decreased level of E-cadherin protein and increased level of $\mathrm{N}$-cadherin protein in Hep3B cells, as suggested by western blot analysis. HCC, hepatocellular carcinoma. ${ }^{*} \mathrm{P}<0.05$.

A

A Control

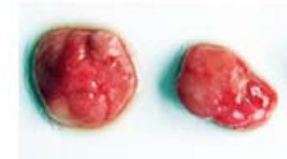
$\underset{\text { miR-3653 }}{\text { mimic }}$
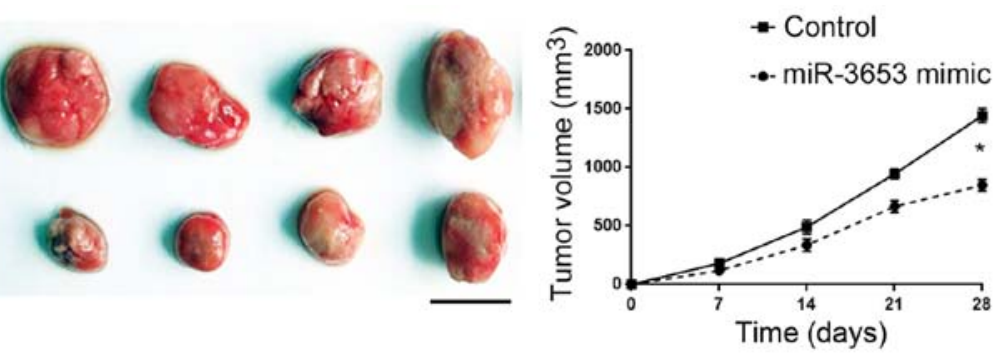

B
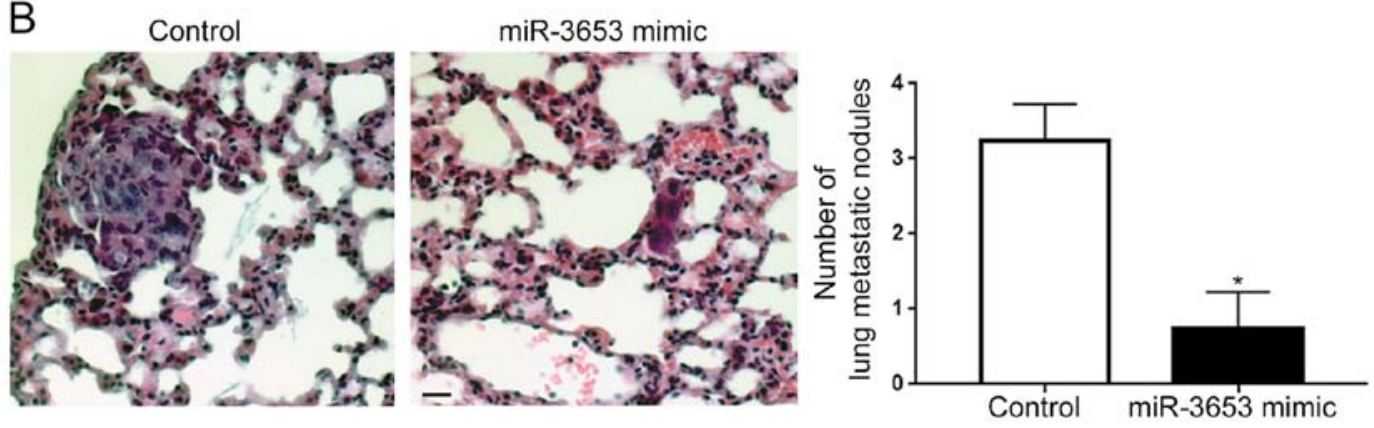

Figure 6. miR-3653 decreases the growth and metastasis of HCCLM3 cells in nude mice. (A) Overexpression of miR-3653 slowed down the growth of HCCLM3 cell-derived tumors in nude mice as suggested by subcutaneous injection experiment. Scale bar, $1 \mathrm{~cm}$. (B) Overexpression of miR-3653 reduced the lung metastatic nodules formed by HCCLM3 cells in nude mice as suggested by tail vein injection experiment. Scale bar, $50 \mu \mathrm{m}$. ${ }^{*} \mathrm{P}<0.05$.

ITGB1 mediates the biological functions of miR-3653 in HCC. Since ITGB1 was confirmed as the downstream target of miR-3653 in HCC, we further explored whether ITGB1 mediates the biological functions of miR-3653 in HCC. qRT-PCR showed that transfection of the ITGB1 vector significantly increased the ITGB1 mRNA in Hep3B cells $(\mathrm{P}<0.05$; Fig. 8A). Western blot analysis showed that transfection of ITGB1 vector in HCCLM3 cells overexpressing miR-3653 significantly increased ITGB1 expression and reversed the increase of E-cadherin and the decrease of $\mathrm{N}$-cadherin caused by miR-3653 overexpression $(\mathrm{P}<0.05$; Fig. 8B). Functionally, forced expression of ITGB1 abrogated the decrease of cell viability and proliferation caused by miR-3653 overexpression ( $\mathrm{P}<0.05$; Fig. $8 \mathrm{C}$ and D). Transwell assay showed that ITGB1 overexpression reversed the decrease of cell migration and invasion induced by miR-3653 overexpression $(\mathrm{P}<0.05$; Fig. 8E).

\section{Discussion}

Hepatocellular carcinoma (HCC) is a fetal disease affecting millions of individuals worldwide. For patients in advanced stages, few effective options are available. The critical 
A

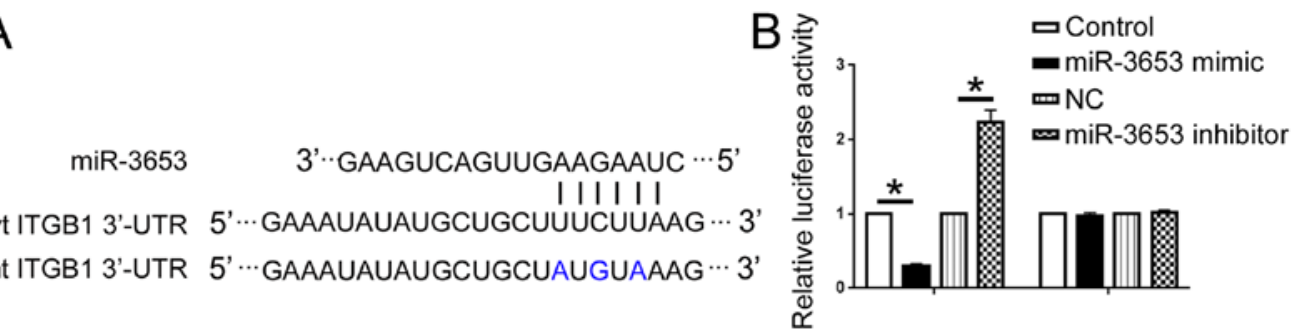
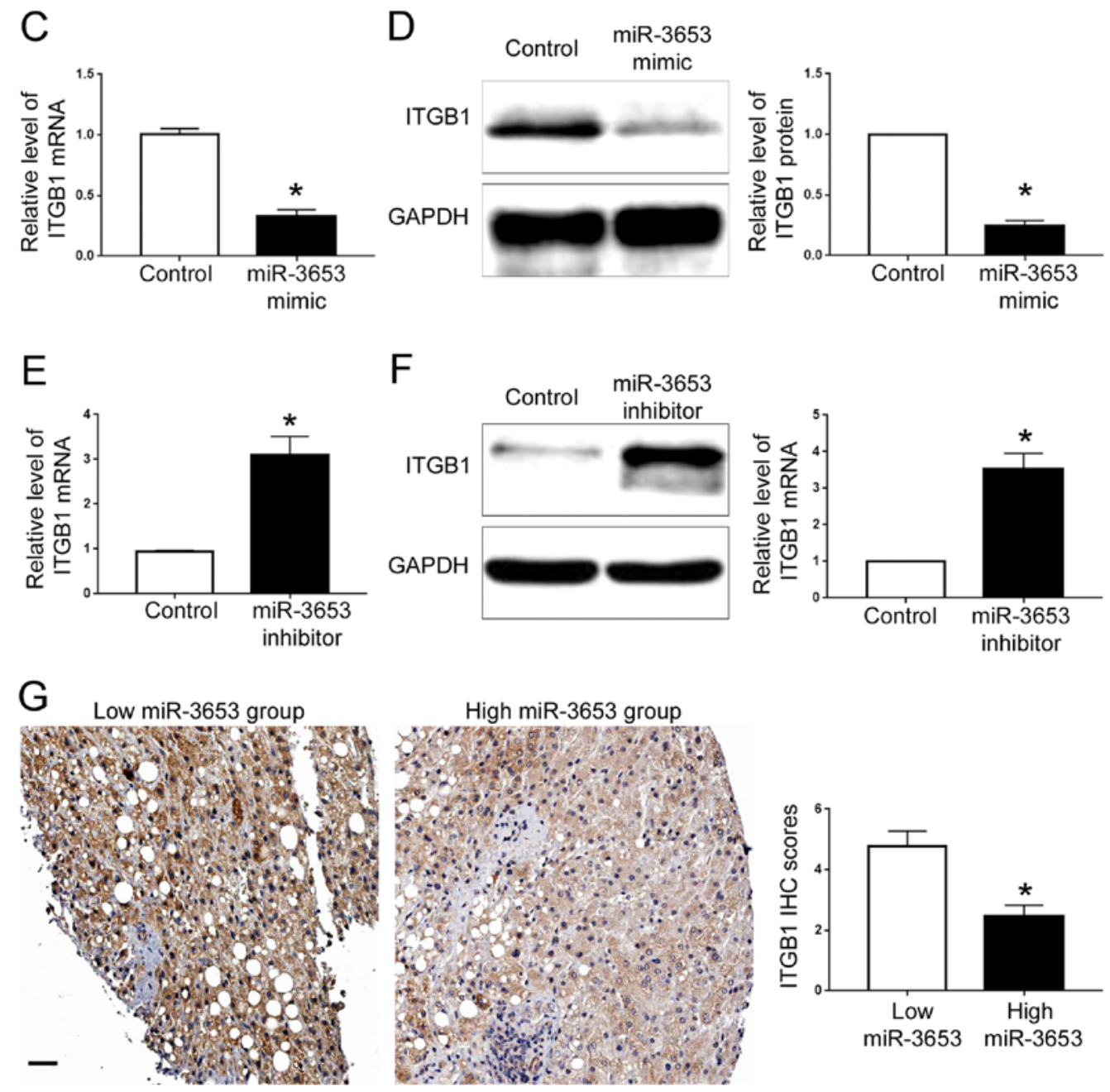

Figure 7. ITGB1 is a direct target of miR-3653 in HCC. (A) miR-3653 was found to contain the complementary sequences mediating the binding between miR-3653 and ITGB1 3'-UTR. (B) Overexpression of miR-3653 decreased the luciferase activity of the wild-type (wt) ITGB1 3'-UTR but had no obvious influence on that of the mutated (mt) ITGB1 3'-UTR. Knockdown of miR-3653 significantly increased the luciferase activity of the wt 3'-UTR of ITGB1 but had no obvious influence on that of the mt 3'-UTR of ITGB1. (C) Overexpression of miR-3653 significantly decreased the mRNA level of ITGB1 in HCCLM3 cells. (D) Overexpression of miR-3653 significantly decreased the protein level of ITGB1 in HCCLM3 cells. (E) Knockdown of miR-3653 significantly increased the mRNA level of ITGB1 in Hep3B cells. (F) Knockdown of miR-3653 significantly increased the protein level of ITGB1 in Hep3B cells. (G) Immunohistochemical (IHC) staining for ITGB1 in HCC tissues with low miR-3653 levels and those with high miR-3653 levels. Scale bar, $100 \mu \mathrm{m}$. HCC, hepatocellular carcinoma; ITGB1; integrin- $\beta 1$. "P<0.05.

molecular mechanisms responsible for HCC progression remain unknown. Previous studies have confirmed that microRNAs (miRNAs) play critical roles in HCC progression $(11,12)$. Numerous cancer-associated miRNAs have been found to affect the growth, metastasis, stem cell formation and drug resistance of HCC cells (13-16). miR-3653 is a novel cancer-associated miRNA and has been found to be aberrantly expressed in different types of human cancers $(3,4)$. However, the expression of miR-3653 in HCC remains unknown. The present study revealed for the first time that
miR-3653 expression is significantly decreased in HCC tissues and cells. Patients with large tumor size or occurrence of metastasis showed a significantly decreased miR-3653 level. Survival analysis demonstrated that a decreased miR-3653 level is correlated with a poor prognosis of HCC patients. These data indicate that miR-3653 acts as a tumor suppressor in $\mathrm{HCC}$.

Uncontrolled growth and local or systemic metastasis are the main reasons for the poor survival of HCC patients. In this study, using gain- and loss-of function experiments, we 

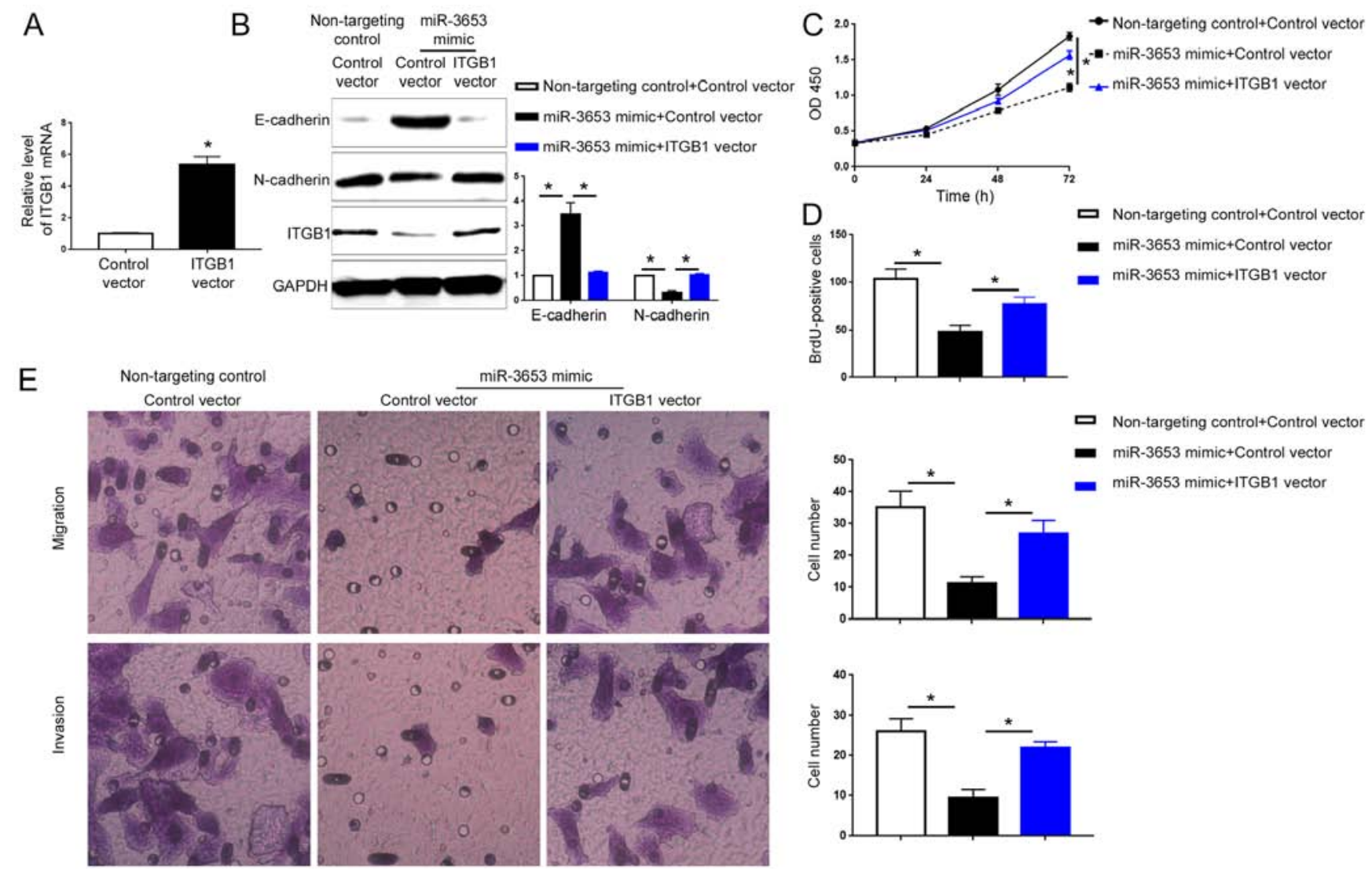

Figure 8. ITGB1 mediates the biological functions of miR-3653 in HCC cells. (A) HCCLM3 cells were transfected with control vector or ITGB1 vector. qRT-PCR showed that the ITGB1 vector effectively increased ITGB1 mRNA in HCCLM3 cells. (B) HCCLM3 cells overexpressing miR-3653 were transfected with the control vector or ITGB1 vector. Transfection of ITGB1 vector restored ITGB1 expression in HCCLM3 cells overexpressing miR-3653, and led to decreased E-cadherin and increased N-cadherin. (C and D) Restoration of ITGB1 reversed the inhibitory effects of miR-3653 overexpression on the cell viability and proliferation of HCCLM3 cells. (E) Restoration of ITGB1 reversed the inhibitory effects of miR-3653 overexpression on the migration and invasion of HCCLM3 cells. Magnification, x40. HCC, hepatocellular carcinoma; ITGB1; integrin- $\beta 1$. " $\mathrm{P}<0.05$.

confirmed that miR-3653 overexpression inhibited the cell viability, proliferation, migration and invasion of HCCLM3 cells while miR-3653 knockdown led to opposite functional consequences. Previous studies demonstrated that EMT is an important mechanism of cancer metastasis. This study found that overexpression of miR-3653 resulted in increased E-cadherin level and decreased $\mathrm{N}$-cadherin expression in HCCLM3 cells while knockdown of miR-3653 led to opposite effects in Hep3B cells. Thus, miR-3653 inhibits the process of EMT of HCC cells. Moreover, this study established a subcutaneous injection model and tail vein injection model to evaluate the influence of miR-3653 on the in vivo growth and metastasis of HCC cells. In vivo assays showed that forced expression of miR-3653 inhibited the growth and metastasis of HCCLM3 cells in nude mice. Taken together, this study demonstrated that miR-3653 inhibited the growth, metastasis and EMT of HCC cells.

ITGB1 functions as an oncogene in different types of human cancers (17-19). It was found to activate the FAK/AKT pathway in cancer cells (20). As for HCC, ITGB1 was found to promote the growth and metastasis of HCC cells (10). In this study, the data of luciferase assay, qRT-PCR and western blot analysis consistently showed that miR-3653 could inhibit the expression of ITGB1 in HCC cells by binding with the 3'-UTR of ITGB1. Rescue experiments confirmed that
ITGB1 overexpression could abrogate the inhibitory effect of miR-3653 on EMT, cell viability, proliferation and metastasis. Thus, ITGB1 is not only a downstream target of miR-3653 but also a functional mediator of miR-3653 in HCC. It is worth noting that ITGB1 was found to be under the control of many other miRNAs including miR-29a (21) and miR-124 (19). These miRNAs were found to be aberrantly expressed in HCC $(22,23)$. Therefore, the expression of ITGB1 in HCC is probably under the control of many different miRNAs.

Collectively, the present study demonstrated that miR-3653 expression is significantly decreased in HCC tissues and cells. Decreased expression of miR-3653 is associated with poor prognosis of HCC patients. miR-3653 was found to inhibit the growth, metastasis and EMT of HCC cells. Moreover, we confirmed for the first time that ITGB1 is a downstream target of miR-3653 and mediates the biological functions of miR-3653 in HCC.

\section{Acknowledgements}

Not applicable.

\section{Funding}

No funding was received. 


\section{Availability of data and materials}

The datasets used during the present study are available from the corresponding author upon reasonable request.

\section{Authors' contributions}

LZ, TZ and ZD acquired the data and created a draft of the manuscript; LZ and ZD prepared the experimental materials and performed the in vitro assays; LZ and LS interpreted the data, performed the statistical analysis and analyzed the results; LZ and LS revised and approved the final version of the manuscript. All authors read and approved the manuscript and agree to be accountable for all aspects of the research in ensuring that the accuracy or integrity of any part of the work are appropriately investigated and resolved.

\section{Ethics approval and consent to participate}

The protocol of the present study was approved by the Institutional Research Ethics Committee of the First Affiliated Hospital of Xinjiang Medical University and informed consent was obtained from every patient enrolled in this study. The protocols regarding the in vivo manipulations were approved by the Animal Care Committee of the First Affiliated Hospital of Xinjiang Medical University.

\section{Patient consent for publication}

Not applicable.

\section{Competing interests}

The authors declare that they have no competing interests.

\section{References}

1. Yates LA, Norbury CJ and Gilbert RJ: The long and short of microRNA. Cell 153: 516-519, 2013.

2. Nelson KM and Weiss GJ: MicroRNAs and cancer: Past, present, and potential future. Mol Cancer Ther 7: 3655-3660, 2008.

3. Lin K, Xu T, He BS, Pan YQ, Sun HL, Peng HX, Hu XX and Wang SK: MicroRNA expression profiles predict progression and clinical outcome in lung adenocarcinoma. Onco Targets Ther 9: 5679-5692, 2016.

4. Wu X, Li S, Xu X, Wu S, Chen R, Jiang Q, Li Y and Xu Y: The potential value of miR-1 and miR-374b as biomarkers for colorectal cancer. Int J Clin Exp Pathol 8: 2840-2851, 2015.

5. El-Serag HB and Rudolph KL: Hepatocellular carcinoma: Epidemiology and molecularcarcinogenesis. Gastroenterology 132: 2557-2576, 2007.

6. Maluccio M and Covey A: Recent progress in understanding, diagnosing, and treating hepatocellular carcinoma. CA Cancer $\mathrm{J}$ Clin 62: 394-399, 2012.

7. Daniel R, Wu Q, Williams V, Clark G, Guruli G and Zehner Z: A panel of MicroRNAs as diagnostic biomarkers for the identification of prostate cancer. Int J Mol Sci 18: pii: E1281, 2017.
8. Livak KJ and Schmittgen TD: Analysis of relative gene expression data using real-time quantitative PCR and the $2^{-\Delta \Delta C_{\mathrm{T}}}$ method. Methods 25: 402-408, 2001.

9. Yilmaz M and Christofori G: EMT, the cytoskeleton, and cancer cell invasion. Cancer Metastasis Rev 28: 15-33, 2009.

10. Zhang YY, Kong LQ, Zhu XD, Cai H, Wang CH, Shi WK, Cao MQ, Li XL, Li KS, Zhang SZ, et al: CD31 regulates metastasis by inducing epithelial-mesenchymal transition in hepatocellular carcinoma via the ITGB1-FAK-Akt signaling pathway. Cancer Lett 429: 29-40, 2018.

11. Gramantieri L, Fornari F, Callegari E, Sabbioni S, Lanza G, Croce CM, Bolondi L and Negrini M: MicroRNA involvement in hepatocellular carcinoma. J Cell Mol Med 12: 2189-2204, 2008.

12. Braconi C, Henry JC, Kogure T, Schmittgen T and Patel T: The role of microRNAs in human liver cancers. Semin Oncol 38: 752-763, 2011.

13. Greenhill C: Hepatocellular carcinoma: New insight into angiogenesis in hepatocellular carcinoma: Involvement of microRNA-26a. Nat Rev Gastroenterol Hepatol 11: 3, 2014.

14. Dou C, Wang Y, Li C, Liu Z, Jia Y, Li Q, Yang W, Yao Y, Liu Q and Tu K: MicroRNA-212 suppresses tumor growth of human hepatocellular carcinoma by targeting FOXA1. Oncotarget 6 : 13216-13228, 2015.

15. Meng F, Henson R, Wehbe-Janek H, Ghoshal K, Jacob ST and Patel T: MicroRNA-21 regulates expression of the PTEN tumor suppressor gene in human hepatocellular cancer. Gastroenterology 133: 647-658, 2007.

16. Zheng F, Liao YJ, Cai MY, Liu YH, Liu TH, Chen SP, Bian XW, Guan XY, Lin MC, Zeng YX, et al: The putative tumour suppressor microRNA-124 modulates hepatocellular carcinoma cell aggressiveness by repressing ROCK2 and EZH2. Gut 61: 278-289, 2012.

17. Kurozumi A, Goto Y, Matsushita R, Fukumoto I, Kato M, Nishikawa R, Sakamoto S, Enokida H, Nakagawa M, Ichikawa T and Seki N: Tumor-suppressive microRNA-223 inhibits cancer cell migration and invasion by targeting ITGA3/ITGB1 signaling in prostate cancer. Cancer Sci 107: 84-94, 2016.

18. Hu C, Ni Z, Li BS, Yong X, Yang X, Zhang JW, Zhang D, Qin Y, Jie MM, Dong H, et al: hTERT promotes the invasion of gastric cancer cells by enhancing FOXO3a ubiquitination and subsequent ITGB1 upregulation. Gut 66: 31-42, 2017.

19. Hunt S, Jones AV, Hinsley EE, Whawell SA and Lambert DW: MicroRNA-124 suppresses oral squamous cell carcinoma motility by targeting ITGB1. FEBS Lett 585: 187-192, 2011.

20. Yang J, Hou Y, Zhou M, Wen S, Zhou J, Xu L, Tang X, Du YE, $\mathrm{Hu} \mathrm{P}$ and Liu M: Twist induces epithelial-mesenchymal transition and cell motility in breast cancer via ITGB1-FAK/ILK signaling axis and its associated downstream network. Int J Biochem Cell Biol 71: 62-71, 2016.

21. He B, Xiao YF, Tang B, Wu YY, Hu CJ, Xie R, Yang X, Yu ST, Dong $\mathrm{H}$, Zhao XY, et al: hTERT mediates gastric cancer metastasis partially through the indirect targeting of ITGB1 by microRNA-29a. Sci Rep 6: 21955, 2016.

22. Furuta M, Kozaki KI, Tanaka S, Arii S, Imoto I and Inazawa J: $m i R-124$ and $m i R-203$ are epigenetically silenced tumor-suppressive microRNAs in hepatocellular carcinoma. Carcinogenesis 31: 766-776, 2010.

23. Zhu XC, Dong QZ, Zhang XF, Deng B, Jia HL, Ye QH, Qin LX and Wu XZ: microRNA-29a suppresses cell proliferation by targeting SPARC in hepatocellular carcinoma. Int J Mol Med 30: 1321-1326, 2012.

This work is licensed under a Creative Commons Attribution-NonCommercial-NoDerivatives 4.0 International (CC BY-NC-ND 4.0) License. 